\section{Asthma-Prävention: vielversprechende Forschungsansätze}

\section{Bei der Asthma-Prävention und -Therapie richten sich die Hoffnungen derzeit vor allem auf den Einsatz verschiedener monoklonaler Antikörper.}

D ie Erfahrung, daß Allergiker besonders anfällig für virale Infektionen sind, findet ihre Erklärung darin, daß eines der während Entzündungsreaktionen vermehrt exprimierten Adhäsionsmoleküle in den Schleimhäuten, nämlich ICAM-1, zugleich Hauptrezeptor für das humane Rhinovirus ist. Virale Infektionen sind nach einer jüngeren Untersuchung mit 80 bis $85 \%$ der Asthma-Exazerbationen bei Kindern assoziiert. Die Viren, so fand man, interagieren mit den Epithelzellen der Atemwege, generieren Zytokine und fördern damit die Eosinophilen-Rekrutierung. Man hofft daher, mit löslichen Adhäsionsmolekülen die Viren abfangen und so ihr Andocken am Schleimhautepithel verhindern zu können.

Monoklonale Antikörper können zirkulierendes $\operatorname{IgE}$ neutralisieren. Durch Bindung an entsprechende Stellen auf dem Immunglobulin verhindern sie dessen Anheftung an die Effektorzelle (Mastzelle oder basophiler Granulozyt). Damit werden alle durch diese Bindung ausgelösten Folgereaktionen, also die Degranulation der Mastzelle und die Mediator-Freisetzung, mit ihren unerwünschten Wirkungen unterbunden. In klinischen Studien vermochten Anti-IgEAntikörper sowohl die Früh- wie Spätphasenreaktion nach Allergenexposition zu modifizieren.

IL-5 nimmt eine zentrale Stellung bei der terminalen Differenzierung, Migration und Aktivierung von Eosinophilen bei der Atemwegsentzündung ein. Spezifisch gegen das Zytokin gerichtete Antikörper, so die Überlegungen, könnten die Rekrutierung dieser Granulozyten verhindern und darüber die Entzündungsreaktion asthmatischer Luftwege verhindern. Tatsächlich ließ sich am Mausmodell demonstrieren, daß die Behandlung mit solchen IL-5-Inhibitoren vor einer Allergen-Provokation die Zahl der Eosinophilen in der bronchoalveolären Lavage und im Lungengewebe herabsetzt. Bei Primaten konnte durch Anwendung dieses Prinzips die Atemwegs-Hyperreaktivität nach Allergen-Provokation gemindert werden. Nach diversen positiv verlaufenen
D ie pathophysiologische Bedeutung der Hausstaubmilbe bei atopischem Ekzem ist zwar umstritten. Bei vielen Patienten haben sich aber Maßnahmen zur Antigenreduktion im Schlafzimmer als vorteilhaft erwiesen. Bekannt ist auch, daß die Applikation des Hausstaubmilben-Antigens Der p1 auf der Haut von Patienten mit atopischem Ekzem $\mathrm{zu}$ einer lokalen Überempfindlichkeitsreaktion vom verzögerten Typ führt. Dies ist die Grundlage der positiven Ergebnisse im Atopie-PatchTest, bei dem Der p1 auf nichtbefallenen Hautarealen eine Reaktion auslöst. Hautbiopsien haben gezeigt, daß das Allergen in die Haut eindringen kann. Sind spezifische IgE-Antikörper vorhanden, ist das Risiko einer Hautreaktion groß.

Die Konsequenz aus diesen Befunden ist nach Ansicht von G. Riley und Mitarbeitern von der Wellington School of Medicine in Neuseeland, die Haut der Patienten in die Maßnahmen
Tierversuchen darf man auf die anstehende Untersuchung monoklonaler Antikörper gegen IL-5 am Menschen gespannt sein.

Beim Asthma als chronischer und auf lange Sicht irreversibler Erkrankung muß Prävention als effektivste Therapie begriffen werden, erklärt W. Busse, Madison/USA. Sowohl monoklonale Antikörper gegen Adhäsionsmoleküle wie solche, die gegen IgE oder IL-5 gerichtet sind, haben nach seiner Ansicht nicht nur theoretisch das Potential, bei akuten Exazerbationen zu intervenieren, sondern könnten sogar eine Rolle bei der primären Prävention spielen.

(wpa)

Busse W: Current research and future needs in allergic rhinitis and asthma. J Allergy Clin Immunol (1998) 101: 424-426.

\title{
Hilft Duschen beim atopischen Ekzem?
}

\section{Morgendliches Duschen führt zu einer Verminderung der Allergenmenge auf der Haut und könnte deshalb bei atopischem Ekzem hilfreich sein.}

zur Allergenreduktion einzubeziehen. Sie empfehlen hierzu morgendliches Duschen.

In einer Untersuchung an 25 gesunden Probanden konnten sie zeigen, daß die Allergenmenge auf der Haut mit der Allergenmenge in den Bettlaken signifikant korreliert. Mit einem handelsüblichen Staubsauger (1000 Watt) wurden in zwei Minuten 3,7 ng Der p1 in 4,5 mg Staub gefunden. Umgerechnet auf den Quadratmeter Hautoberfläche sind dies 2,35 ng Der p1. Das ist zwar nur die Hälfte von dem, was im Atopie-PatchTest auf einen Quadratzentimeter $(4,9 \mathrm{ng})$ Haut aufgetragen wird. Dennoch ist laut Riley nicht auszuschließen, daß in Einzelfällen Hausstaubmilben an der Exazerbation eines Ekzems beteiligt sein könnten. Untersuchungen, die dies bestätigen, fehlen jedoch noch.

(rme)

Riley G et al.: House-dust mite antigen on skin and sheets Lancet (1998); 351: 649-650. 